\title{
Visibility Driven Hierarchical Radiosity
}

\author{
Frédo Durand, George Drettakis and Claude Puech
}

iMAGIS*- GRAVIR/IMAG - INRIA

\section{Introduction and Previous Work}

Hierarchical radiosity (HR) simulations rapidly compute light exchanges in a given scene by subdividing the original objects to create an adaptive mesh representing light variations and shadows. Previous mesh subdivision strategies still suffer however from the limitations of regular (quadtree) subdivision. Discontinuity meshing solutions are usually limited to primary illumination and become intractable for more than two or three light sources. Visibility calculation is still done largely by ray-casting approximations which can often produce erroneous results.

In this abstract, we present a novel Visibility Driven hierarchical radiosity algorithm, by extending the Visibility Skeleton [DDP97]. Our approach uses the extended Skeleton to compute accurate visibility at vertices, to insert important discontinuities and to effect an efficient refinement strategy. We use hierarchical triangulations and introduce a novel push-pull procedure for accurate display.

The most significant related work includes that of Lischinski et al [LTG93] which combines discontinuity meshing and HR. Discontinuity meshing is performed for a small number of direct lights, and a second pass is used to accurately compute visibility and illumination at vertices. Our visibility-driven HR algorithm is also related to Wavelet radiosity [GSCH93] and to the work on perceptually based refinement [GH97].

\section{Extended Skeleton}

The Visibility Skeleton is an encoding of all visibility events in a scene, using a graph structure. The nodes of the graph are extremal stabbing lines and the arcs critical line swaths which allow the calculation of the exact visible contour of every polygon from every scene vertex [DDP97]. We have extended this structure, hereby allowing the calculation of views at vertices resulting from the subdivision of original scene polygons required by the radiosity algorithm. We have also replaced the quadratic table representation of arcs by linking mutually visible objects. These links are also used in the lighting solution. All polygon-vertex form-factors (including those for indirect light transfers) are thus computed exactly.

\section{Embedded Triangulation Hierarchies for Accurate Hierarchical Radiosity}

To accurately represent radiosity across surfaces, especially for the very irregular discontinuities created by shadow borders, we have chosen to use hierarchical Delaunay triangulations (similar to [DP95]). Instead of computing radiosity at patch centers, we gather light at each vertex. This requires a new push procedure, which allows the contribution of a source to a vertex at a certain level to be pushed to the corresponding children. This is done by storing radiosity differences between the approximation at the higher level and the value at the current level. During the push, a vertex value at the current level is simply the sum of the stored difference and the interpolated value of the higher level at this vertex.

*iMAGIS is a joint research project of CNRS/INRIA/UJF/INPG. iMAGIS/GRAVIR, BP 53, F-38041 Grenoble Cedex 09 France. Email: Fredo.Durand|George.Drettakis|Claude.Puech\}@imag.fr http://www-imagis.imag.fr
The result of this approach is a high quality, continuous linear representation of radiosity at the leaves of the mesh, obviating the need for a second pass for display.

\section{Visibility Driven Hierarchical Radiosity}

We maintain two link types: vertex-polygon links for gathering, and polygon-polygon links used to determine if a light transfer should be refined. The two link types are subdivided simultaneously resulting in a consistent representation of light transfer.

To refine the scene polygons we use the visibility information of the Skeleton. For a given receiver, we examine all its polygonpolygon links and the contained visibility events. The corresponding discontinuities are ranked using their perceptually based importance, and only a certain percentage are actually inserted into the receiver Delaunay triangulation. It is important to note that this operation takes into account all visibility interactions, including those for indirect illumination links. We also subdivide at the maximum of the unoccluded light source radiosity for large receivers. Subdivision is based on the difference between exact and interpolated values for the unoccluded case, and an estimation of shadow importance elsewhere.

\subsection{Results}

The following figure shows the result of our implementation for a scene containing 10 light sources and 486 polygons, and a scene of 534 polygons with mainly indirect lighting due to the lefthand table and the bed. The mesh adapts to the combined shadow effects automatically, resulting in high quality illumination. The images were rendered directly using graphics hardware and did not require a local or final gather pass.
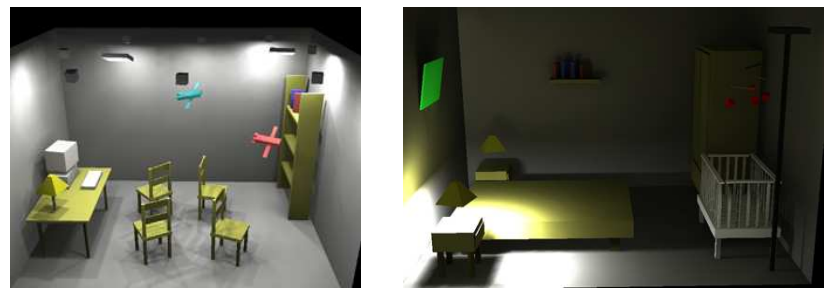

Figure 1: (a) Skeleton construction took 2 min 23s.; meshing/lighting step took $8 \mathrm{~min}$. (b) Skeleton construction took 4min 12s; meshing/lighting step took $7 \mathrm{~min}$.

\section{References}

[DDP97] F. Durand, G. Drettakis, and C. Puech. The visibility skeleton: A powerful and efficient multi-purpose global visibility tool. In Turner Whitted, editor, ACM SIGGRAPH Annual Conf. Series, pp. 89-100, August 1997.

[DP95] L. De Floriani and E. Puppo. Hierarchical triangulation for multiresolution surface description geometric design. ACM Trans. on Graphics, 14(4):363-411, October 1995.

[GH97] S. Gibson and R. J. Hubbold. Perceptually-driven radiosity. Computer Graphics Forum, 16(2):129-141, 1997.

[GSCH93] S. J. Gortler, P. Schroder, M. F. Cohen, and P. Hanrahan. Wavelet radiosity. In Computer Graphics Proc., ACM SIGGRAPH Annual Conference Series, 1993, pages 221-230, 1993.

[LTG93] D. Lischinski, F. Tampieri, and D. P. Greenberg. Combining hierarchical radiosity and discontinuity meshing. In Computer Graphics Proc., ACM SIGGRAPH Annual Conference Series, 1993, pages 199-208, 1993. 